\title{
Reynolds number scaling of velocity increments in isotropic turbulence
}

\author{
Kartik P. Iyer* \\ Department of Physics and INFN, University of Rome Tor Vergata, Rome 00133, Italy \\ and Department of Mechanical Engineering, New York University, New York 11201, USA \\ Katepalli R. Sreenivasan \\ Departments of Physics and Mechanical Engineering and the Courant Institute of Mathematical Sciences, \\ New York University, New York 11201, USA \\ P. K. Yeung \\ Schools of Aerospace Engineering and Mechanical Engineering, Georgia Institute of Technology, Atlanta, Georgia 30332, USA
}

(Received 30 October 2016; revised manuscript received 17 January 2017; published 10 February 2017)

\begin{abstract}
Using the largest database of isotropic turbulence available to date, generated by the direct numerical simulation (DNS) of the Navier-Stokes equations on an $8192^{3}$ periodic box, we show that the longitudinal and transverse velocity increments scale identically in the inertial range. By examining the DNS data at several Reynolds numbers, we infer that the contradictory results of the past on the inertial-range universality are artifacts of low Reynolds number and residual anisotropy. We further show that both longitudinal and transverse velocity increments scale on locally averaged dissipation rate, just as postulated by Kolmogorov's refined similarity hypothesis, and that, in isotropic turbulence, a single independent scaling adequately describes fluid turbulence in the inertial range.
\end{abstract}

DOI: 10.1103/PhysRevE.95.021101

\section{INTRODUCTION}

This Rapid Communication resolves the previously contradictory results on inertial-range universality, to be described immediately below, of two orthogonal velocity increments in isotropic turbulence. Velocity increments are differences of velocities between two spatial positions separated by a fixed distance, and are of fundamental importance in turbulence research [1-3]. In isotropic turbulence, velocity increments in parallel and perpendicular directions to the separation distance $r$, known as the longitudinal $\left(\delta u_{r}\right)$ and transverse $\left(\delta v_{r}\right)$ increments, respectively, describe the velocity increment statistics completely. These statistics are usually in the form of moments of $\delta u_{r}$ and $\delta v_{r}$, and are known, respectively, as the longitudinal and transverse structure functions. The order of the structure function is the moment order of the velocity increments. The power-law scaling of both the longitudinal and transverse structure functions with respect to $r$ should be the same for inertial-range universality to hold, where the inertial range is the range of separation distances $r$ which are small compared to the large scale of turbulence but large compared to the viscous cut-off scale. The scaling exponents for second-order structure functions of $\delta u_{r}$ and $\delta v_{r}$ are indeed the same, but higher-order quantities can scale differently in principle [4], so there is no a priori assurance of principle that universality holds. The majority of the empirical

\section{*kki2@nyu.edu}

Published by the American Physical Society under the terms of the Creative Commons Attribution 4.0 International license. Further distribution of this work must maintain attribution to the author(s) and the published article's title, journal citation, and DOI. evidence available, both experimental and numerical, shows that longitudinal and transverse structure functions indeed scale differently at higher orders [5-13], though some studies suggest otherwise $[14,15]$. Predicated on the consensus that the longitudinal and transverse structure functions scale differently, it was argued in Ref. [8] that the longitudinal increments are closely associated with the energy dissipation, while the transverse increments are linked more closely to enstrophy (squared turbulent vorticity). This argument seemed eminently plausible at the time because the evidence then available also suggested that, while the mean values of dissipation and enstrophy were equal, they differed in higher-order moments. Thus, theoretical arguments [16-18] which have advanced the view that the two types of structure functions, on the one hand, and energy dissipation and enstrophy, on the other, must scale similarly have been without clear empirical support. Here, we show, using the direct numerical simulation (DNS) data at a variety of Reynolds numbers including the largest simulations available to date, on a box of $8192^{3}$ grid points corresponding to a Taylor-scale Reynolds number $R_{\lambda}$ of 1300 [19], that the theoretical arguments are essentially correct, and that previously observed differences are artifacts of low Reynolds number, remnant shear, etc. In conjunction, we show that the so-called refined similarity hypothesis of Kolmogorov [20], holds for both the longitudinal and transverse increments in the inertial range. This hypothesis links the inertial range dynamics with that of the dissipative range, which is where the energy dissipation essentially occurs.

To be specific, we provide the following definitions before proceeding further. Let $\epsilon=\frac{v}{2}\left(\partial u_{i} / \partial x_{j}+\partial u_{j} / \partial x_{i}\right)^{2}$ denote the turbulent energy dissipation rate per unit mass, where $v$ is the kinematic viscosity of the fluid. The dissipation scale $\eta=$ $\left(v^{3} /\langle\epsilon\rangle\right)^{1 / 4}$, where $\langle\cdot\rangle$ denotes a suitable average. The longitudinal velocity increment is defined as $\delta u_{r} \equiv u(x+r)-u(x)$, 
where the velocity component $u$ and the separation distance $r$ are both in the same direction, $x$. The transverse velocity increment, $\delta v_{r} \equiv v(x+r)-v(x)$, where the separation distance $r$ (still in the direction $x$ ) is normal to the velocity component $v$. Denote the $p$ th-order longitudinal and transverse structure functions as $S_{L}^{p}(r) \equiv\left\langle\left(\delta u_{r}\right)^{p}\right\rangle$ and $S_{T}^{p}(r) \equiv\left\langle\left(\delta v_{r}\right)^{p}\right\rangle$, respectively. All odd moments of $\delta v_{r}$ vanish in isotropic turbulence (but not those of $\delta u_{r}$ ). Assume, following the spirit of Kolmogorov [1], that $S_{L}^{p}(r) \sim r^{\zeta_{L}^{p}}$ and $S_{T}^{p}(r) \sim r^{\zeta_{T}^{p}}$. Since $\zeta_{L}^{2}=\zeta_{T}^{2}$ under isotropy, we aim first to obtain $\zeta_{T}^{p}$ and $\zeta_{L}^{p}$ for $p \geqslant 4$. We obtain scaling exponents of longitudinal and transverse structure functions, $\zeta_{L}^{p}$ and $\zeta_{T}^{p}$, respectively, from a large DNS database of isotropic turbulence in a periodic box, spanning a wide range of Reynolds numbers [21], the largest of them being on a grid size of $8192^{3}$, corresponding to a Taylor-scale Reynolds number $R_{\lambda}$ of 1300 [19]. A statistically steady state is obtained by forcing the low Fourier modes. For details see Refs. [19,22] and the references cited therein. Averages over multiple large-eddy turnover times were used in the analysis.

\section{RESULTS AND DISCUSSION}

In locally isotropic turbulence, the following relations must hold at scale $r$ :

$$
\begin{gathered}
\left\langle\delta v_{r}^{2}\right\rangle=\left\langle\delta u_{r}^{2}\right\rangle+\frac{r}{2} \frac{d}{d r}\left\langle\delta u_{r}^{2}\right\rangle, \\
\left\langle\delta v_{r}^{3}\right\rangle=0
\end{gathered}
$$

Figure 1 examines Eqs. (1) and (2) at $R_{\lambda}=140$ and 1300, as a function of scale separation. At a given grid resolution the residual anisotropy, due to factors such as finite box size and cubic grid geometry, increases with scale $r$. However, at higher $R_{\lambda}$, there exists a wider range of intermediate scales, $\eta / L_{0} \ll r / L_{0} \ll 1$, that is nearly devoid of such effects.

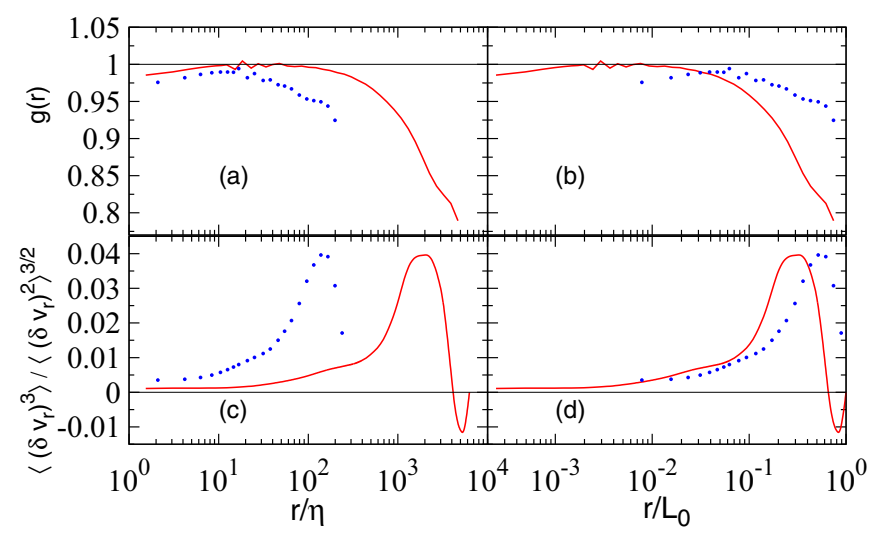

FIG. 1. Isotropy tests at $R_{\lambda}=140$ (dotted line) and $R_{\lambda}=1300$ (solid line), as functions of scale separation $r$ in a cubic box with edge length $2 L_{0}$. Panels (a) and (b) show $g(r)$, the right-hand side of Eq. (1) divided by the left-hand side of the same, against $r / \eta$ and $r / L_{0}$, respectively. Horizontal line at unity is the isotropic value, $g(r)=1$, for reference. Panels (c) and (d) show the skewness of the transverse structure function against $r / \eta$ and $r / L_{0}$, respectively [Eq. (2)]. The reference line at zero shows the exact isotropic value.

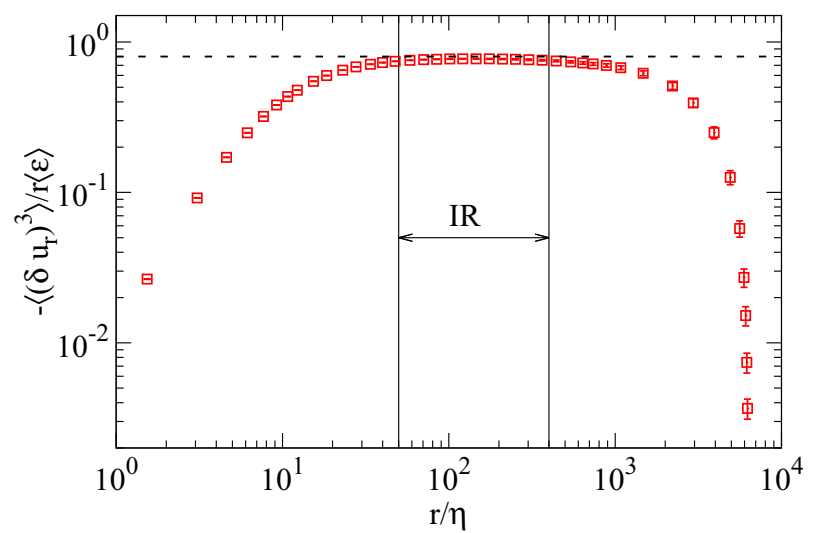

FIG. 2. Normalized third-order longitudinal velocity structure function at $8192^{3}, R_{\lambda}=1300$. The dashed horizontal line corresponds to the plateau at $4 / 5$. Vertical lines demarcate inertial range $50<$ $r / \eta<400$, considered as the range within $5 \%$ deviation from $4 / 5$. Error bars indicate $95 \%$ confidence intervals.

The normalized third-order longitudinal velocity structure function $S_{L}^{3}(r) / r\langle\epsilon\rangle$ is used to determine the extent of the inertial range: it is the range of scales for which $-S_{L}^{3}(r) / r\langle\epsilon\rangle$ is equal to the exact theoretical value of $4 / 5$ [2]. This quantity is plotted in Fig. 2 for the $8192^{3}$ simulation (corresponding to $R_{\lambda}=1300$ ), as a function of the spatial separation, $r$. Denoting the inertial range pragmatically as the scale range within which the exact value of $4 / 5$ is satisfied to better than an accuracy of $5 \%$, we can see from the figure that it corresponds to $50<$ $r / \eta<400$, approximately a decade in extent.

Figure 3 shows the local slopes of the power-law scaling relations, in the inertial range (and to either side of it), computed from the $8192^{3}$ simulation for orders $p=4,6$, and 8. Slopes for both longitudinal and transverse structure functions are shown. It is evident from the figure that they are essentially the same in the inertial range, for each given $p$, at least for $p$ up to 8 .

The longitudinal and transverse scaling exponents obtained from least-squares fits at two different Reynolds numbers, in

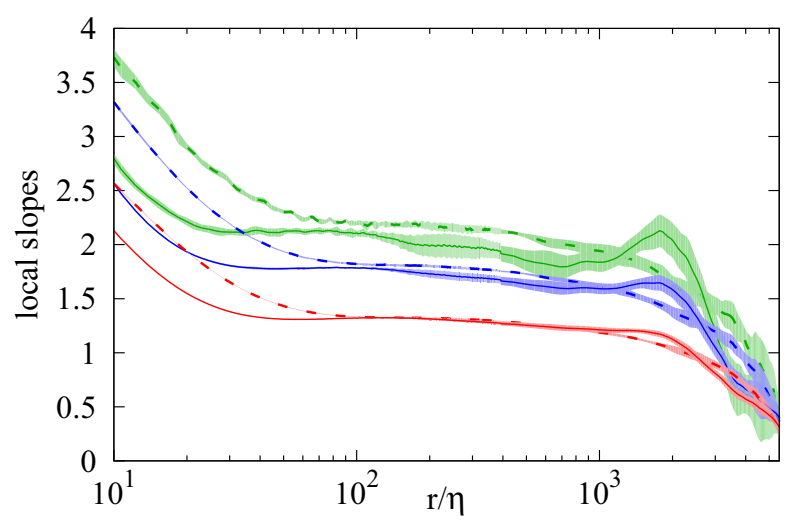

FIG. 3. Inertial range local slopes at $R_{\lambda}=1300$ as a function of spatial separation. Dashed and solid lines correspond to $S_{L}^{p}(r)$ and $S_{T}^{p}(r)$, respectively. Colors red, blue, and green correspond to orders $p=4,6$, and 8 , respectively. Corresponding shaded regions denote $95 \%$ confidence intervals. 
TABLE I. Longitudinal $\left(\varsigma_{L}^{p}\right)$ and transverse $\left(\varsigma_{T}^{p}\right)$ inertial range exponents from least-squares fits at $R_{\lambda}=1300$ and 240. Error bars indicate $95 \%$ confidence intervals. The exponents are computed in the range $r / \eta \in[50,400]$ (see Fig. 3).

\begin{tabular}{cccccc}
\hline \hline & \multicolumn{2}{c}{$R_{\lambda}=1300$} & & \multicolumn{2}{c}{$R_{\lambda}=240$} \\
\cline { 2 - 3 } \cline { 5 - 6 }$p$ & $\varsigma_{L}^{p}$ & $\varsigma_{T}^{p}$ & & $\varsigma_{L}^{p}$ & $\varsigma_{T}^{p}$ \\
\hline 2 & $0.72 \pm 0.002$ & $0.72 \pm 0.003$ & & $0.70 \pm 0.012$ & $0.69 \pm 0.014$ \\
4 & $1.30 \pm 0.005$ & $1.30 \pm 0.010$ & & $1.27 \pm 0.024$ & $1.22 \pm 0.024$ \\
6 & $1.78 \pm 0.010$ & $1.76 \pm 0.015$ & & $1.70 \pm 0.038$ & $1.61 \pm 0.045$ \\
8 & $2.18 \pm 0.021$ & $2.12 \pm 0.020$ & & $2.06 \pm 0.072$ & $1.83 \pm 0.091$ \\
\hline \hline
\end{tabular}

the respective inertial ranges, are given in Table I for orders $p=2-8$. The error bars have been obtained from the temporal variations in the fitted slopes from independent stationary state snapshots. The differences between $\varsigma_{L}^{p}$ and $\varsigma_{T}^{p}$ shrink as we go to higher Reynolds numbers, indicating that the mismatch between the exponents at lower $R_{\lambda}$ is the result of a superposition of leading and subleading power laws with coinciding exponents between longitudinal and transverse statistics, but with different prefactors.

To make this point even clearer, one can plot the quantity $\left\langle\left(\delta v_{r}\right)^{p}\right\rangle$ against $\left\langle\left(\delta u_{r}\right)^{p}\right\rangle$, parametrized by various values of $r$. If the slope of such graphs is unity in the inertial range, we can say that the two families of structure functions scale the same. The corresponding plots are shown in Fig. 4, for $p=6$ and $p=8$, for two different Reynolds numbers. Inertial ranges have been identified in the two graphs by the (O) symbol. We find that at the low Reynolds number of $R_{\lambda}=240$, $\left\langle\left(\delta v_{r}\right)^{6}\right\rangle \sim\left\langle\left(\delta u_{r}\right)^{6}\right\rangle^{0.87}$ and $\left\langle\left(\delta v_{r}\right)^{8}\right\rangle \sim\left\langle\left(\delta u_{r}\right)^{8}\right\rangle^{0.82}$, showing that the transverse structure functions have smaller scaling exponents than longitudinal ones. In contrast, for $R_{\lambda}=1300$, we have $\left\langle\left(\delta v_{r}\right)^{6}\right\rangle \sim\left\langle\left(\delta u_{r}\right)^{6}\right\rangle^{0.99}$ and $\left\langle\left(\delta v_{r}\right)^{8}\right\rangle \sim\left\langle\left(\delta u_{r}\right)^{8}\right\rangle^{0.97}$, showing that the two exponents are almost identical. It appears clear that the scaling exponents of the longitudinal and transverse structure functions approach each other as the Reynolds number increases.

To see how fast $\zeta_{L}^{p}$ and $\zeta_{T}^{p}$ approach each other with increasing Reynolds number, we plot the ratio $\zeta_{T}^{p} / \zeta_{L}^{p}$ against $R_{\lambda}$ in Fig. 5. The scaling exponents, $\zeta_{L}^{p}$ and $\zeta_{T}^{p}$, are determined by least-square fits within the inertial range, as illustrated in Fig. 4. These results demonstrate that $\zeta_{L}^{p}$ and $\zeta_{T}^{p}$ approach each other as the Reynolds number increases, and that the differences between them, observed previously, must be attributed to the finite Reynolds number effect. This is the first major conclusion of the Rapid Communication.

An additional supporting comment is useful. The use of local slopes or the extended self-similarity (ESS) technique [23] yields very similar results. In the ESS method, the scaling exponents are determined by plotting various orders of transverse and longitudinal structure functions against their respective second-order moments and determining their slopes in the inertial range.

We now turn to the so-called refined similarity hypothesis (RSH) of Kolmogorov [20], which links statistically the fluctuation of the velocity increments of a known scale, say $r$, in the inertial range to the dissipation fluctuations averaged over a volume of $O\left(r^{3}\right)$. That is, the vector velocity increment

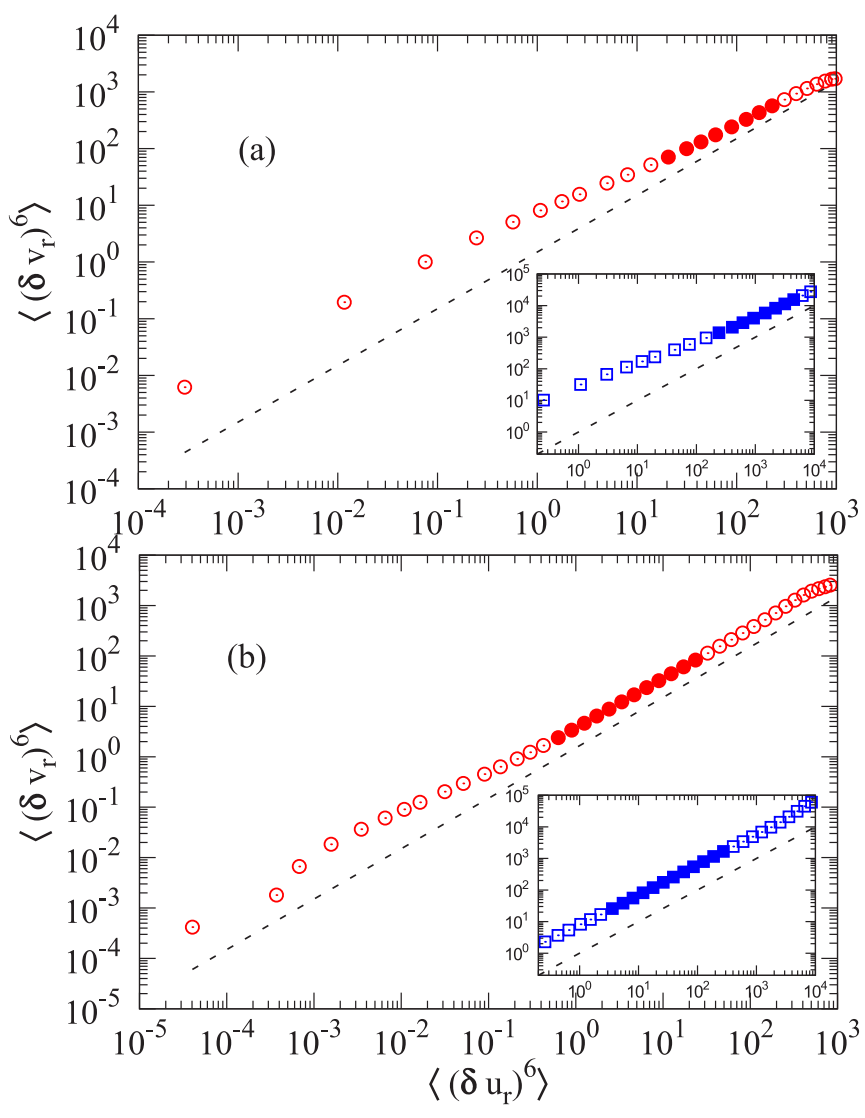

FIG. 4. Relative scaling of transverse structure function against longitudinal structure function at two different Reynolds numbers. Plotted against $\left\langle\left(\delta u_{r}\right)^{6}\right\rangle$ is $\left\langle\left(\delta v_{r}\right)^{6}\right\rangle$ at (a) $R_{\lambda}=240$ and (b) $R_{\lambda}=1300$. The (๑) points are for $r$ in the inertial range, while $(\odot)$ correspond to $r$ outside this range. The dashed line is for $\left\langle\left(\delta v_{r}\right)^{6}\right\rangle \sim\left\langle\left(\delta u_{r}\right)^{6}\right\rangle$. The insets show similar results for the eighth-order structure functions, with the inertial range data highlighted by ( $\square$ ).

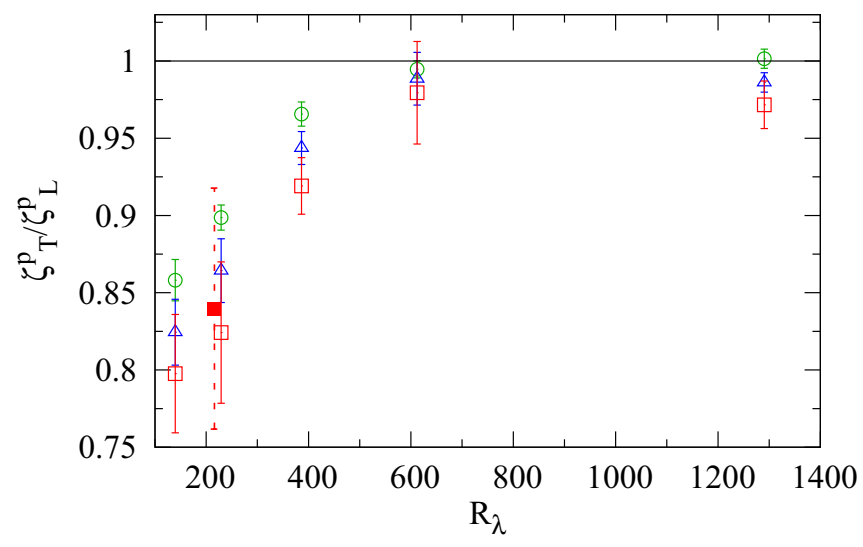

FIG. 5. Ratio of inertial range exponents of $S_{T}^{p}(r)$ and $S_{L}^{p}(r)$ as a function of the Taylor-scale Reynolds number. Symbols $(\circ),(\triangle)$, and $(\square)$ correspond to orders $p=4,6$, and 8 , respectively. The horizontal line at unity is for reference. Solid vertical lines indicate 95\% confidence intervals. The corresponding result of Ref. [8] for $p=8$ ( $\square$ ) with error bars, shown by a dashed vertical line, is given for comparison. 
$[\Delta \mathbf{u}(\mathbf{r})]$ at separation vector $\mathbf{r}$ is statistically related to the three-dimensional average of dissipation at scale $|\mathbf{r}|=r$, $\epsilon_{r} \equiv \int_{V(r)} \epsilon d V$, with the "local" averaging performed over the volume $V(r) \sim O\left(r^{3}\right)$ [20,24], through the relation

$$
\Delta \mathbf{u}(\mathbf{r}) \equiv \mathbf{V}(\mathbf{r})\left(r \epsilon_{r}\right)^{1 / 3} \text {, }
$$

where the dimensionless velocity vector $\mathbf{V}(\mathbf{r})$ is independent of $\epsilon_{r}$ as well as $r$ in the inertial range, and hence universal [20].

It is convenient to rewrite Eq. (3) in terms of two scalar functions $V_{L}$ and $V_{T}$ as

$$
\begin{gathered}
\delta u_{r}=V_{L}\left(r \epsilon_{r}\right)^{1 / 3}, \\
\delta v_{r}=V_{T}\left(r \epsilon_{r}\right)^{1 / 3} .
\end{gathered}
$$

When $p$ is odd, isotropy stipulates that $\left\langle V_{T}^{p}\right\rangle=0$. However, since $\left\langle V_{L}^{p}\right\rangle \neq 0$ for odd $p(>1)$, in the inertial range, it necessarily follows that the functions $V_{L}$ and $V_{T}$ will themselves be different $\left(\delta u_{r}\right.$ is skewed and $\delta v_{r}$ is not) for any given $r \ll L$. So the focus of universality is that $V_{L}$ and $V_{T}$ will both statistically asymptote to independent functions, though different from one another in the inertial range. The universality of $\mathbf{V}(\mathbf{r})$ is a direct consequence of the fact that the longitudinal and transverse structure functions have the same scaling in the inertial range. It then follows naturally that a rigorous examination of the universality of $V_{L}$ and $V_{T}$ serves as useful checks for the result that $\zeta_{L}^{p}=\zeta_{T}^{p}$ at all even $p$, in the inertial range.

There is a second point to be made. If we had followed the ideas of Ref. [8] that the transverse velocity is closely connected to enstrophy, the logical consequence would be to replace the locally averaged energy dissipation in Eq. (5) by the locally averaged enstrophy. However, it has been shown in Ref. [19] that the probability density functions (PDFs) of the energy dissipation and enstrophy are the same for large amplitudes at high Reynolds numbers (while this is not true at low Reynolds numbers considered in Ref. [8]). It thus appears logical to believe that the locally averaged dissipation ought to appear in both Eq. (4) and Eq. (5). Our results verify this assertion as well.

Past verifications of RSH have focused, largely for reasons of convenience, on longitudinal velocity increments [22,25-30]. If $\left\langle\epsilon_{r}{ }^{p}\right\rangle \sim r^{\tau_{p}}$ in the inertial range, it follows from Eq. (4) that

$$
\zeta_{L}^{p}=\frac{p}{3}+\tau_{p / 3}
$$

Now, because $\delta u_{r}$ and $\delta v_{r}$ both scale the same way (i.e., $\zeta_{L}^{p}$ and $\zeta_{T}^{p}$ are the same for even orders), the following relation between $\zeta_{T}^{p}$ and $\tau_{p / 3}$ should also hold good for any even $p$ in the inertial range, so we can write

$$
\zeta_{T}^{p}=\frac{p}{3}+\tau_{p / 3}
$$

We first address the universality of $V_{L}$ and $V_{T}$. In Fig. 6, we plot the PDF of $V_{L}$ and $V_{T}$ for $r$ in the inertial range at $R_{\lambda}=1300$. The respective PDFs of $V_{L}$ and $V_{T}$ collapse for all separations in the inertial range indicating that they are independent of $r$ and $\epsilon_{r}$, as expected from arguments of universality. The conclusion from Fig. 6 is that K62 holds for both longitudinal and transverse increments in the inertial range. As already mentioned, the main difference between the

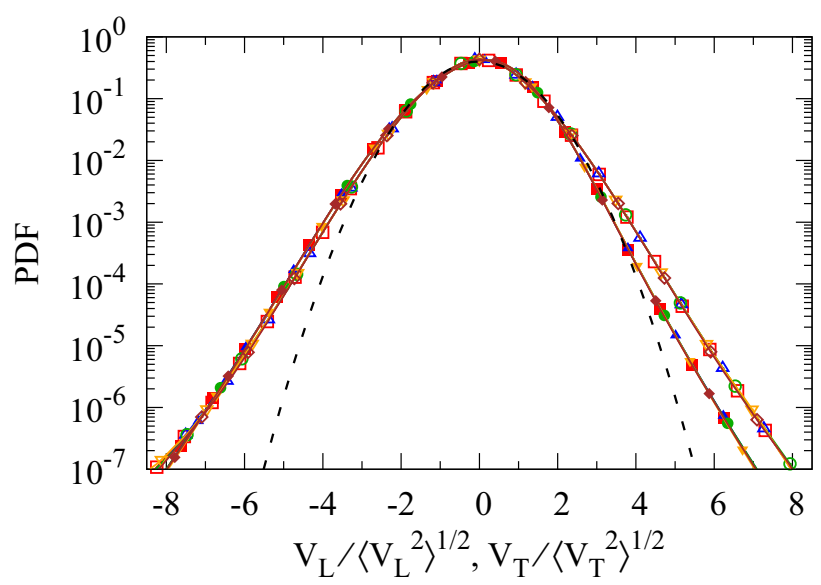

FIG. 6. Normalized PDF of $V_{L}$ and $V_{T}$ at $R_{\lambda}=1300$ for various inertial range separations. Curves with filled symbols correspond to $V_{L}$ for different values of $r:(\nabla) r / \eta=99$, ( $\left.\square\right) r / \eta=118$, ( $)$ $r / \eta=143,(\bigcirc) r / \eta=170,(\nabla) r / \eta=206$. Corresponding curves for $V_{T}$ given by open symbols. The dashed line shows the standard Gaussian for reference.

PDFs of $V_{L}$ and $V_{T}$ is that the former is negatively skewed with $\left\langle V_{L}^{3}\right\rangle=-4 / 5$ [20], while the latter is symmetric around the origin with zero skewness.

Figure 7(a) shows the joint probability density function (JPDF) of $V_{L}$ and $V_{T}$ at an inertial range separation for $R_{\lambda}=$ 1300; (b) and (c) of the figure show slivers of the JPDF to reveal two distinct cross sections.

It is easily seen that the PDFs of $V_{L}$ and $V_{T}$ do not have particularly extended tails, and are hence nonintermittent. It is thus far easier to work with the normalized velocity increments $V_{L}$ and $V_{T}$ statistically than with the velocity increments themselves which are highly intermittent and extremely sensitive to finite sampling effects $[3,5]$.

Since it is now clear that $V_{L}$ and $V_{T}$ are both independent of $r$ and $\epsilon_{r}$ (and of each other), it follows from Eqs. (6) and

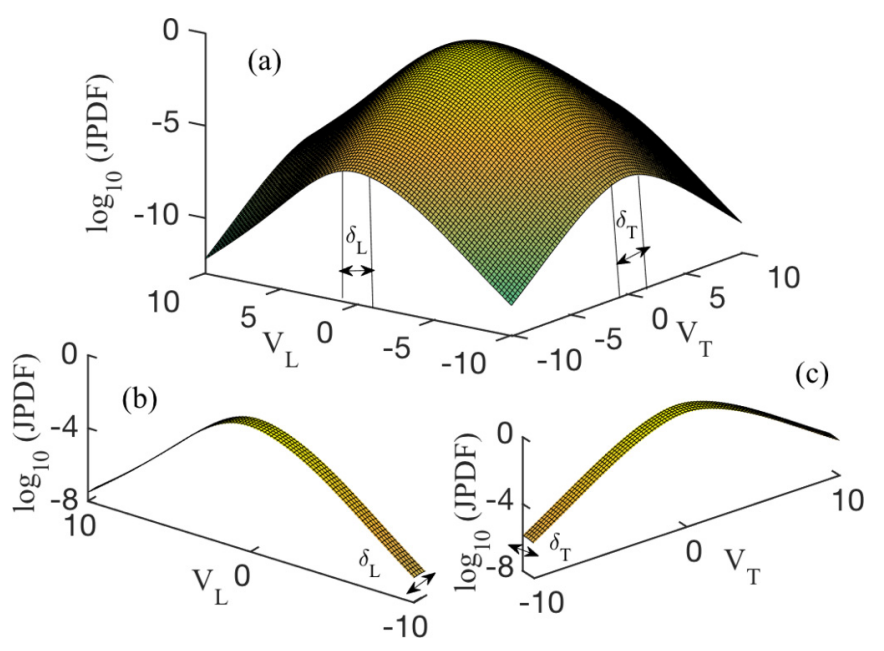

FIG. 7. Probability distribution of the dimensionless relative velocity $\mathbf{V}(\mathbf{r})$ at inertial range separation $r / \eta=100, R_{\lambda}=1300$. (a) Logarithm of the JPDF of $V_{L}$ and $V_{T}$. Slivers of width $\delta_{L}$ and $\delta_{T}$ of the JPDF along $V_{L}$ and $V_{T}$ (marked by vertical lines) are shown in (b) and (c) to reveal the asymmetric and symmetric parts, respectively. 


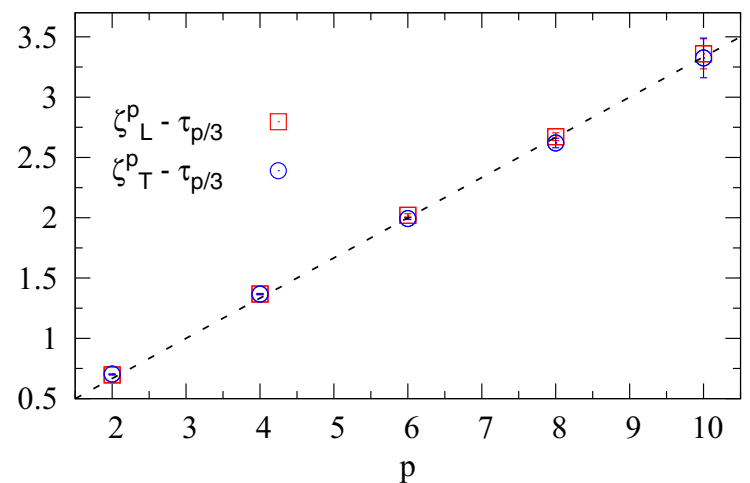

FIG. 8. Verification of the RSH scaling relations [Eqs. (6) and (7)] at $R_{\lambda}=1300$. Symbols ( $\square$ ) and (o) correspond to the longitudinal and transverse cases, respectively. If RSH is correct, the measured differences between $\zeta_{L}^{p}$ and $\tau_{p / 3}$ and that between $\zeta_{T}^{p}$ and $\tau_{p / 3}$ for various values of $p$ must equal $p / 3$, which is the dashed line. The correspondence is very good. Vertical bars indicate the extent of $95 \%$ confidence intervals (which are significant mainly at higher orders).

(7), that $\zeta_{L}^{p}-\tau_{p / 3}$ and $\zeta_{T}^{p}-\tau_{p / 3}$ must both be equal to $p / 3$. In Fig. 8 we verify this expectation from numerical results for $p$ up to 10 . The exponents $\tau_{p / 3}$ of the three-dimensional local averages of dissipation are obtained from least-squares fits in the inertial range. With increasing $p$, both $\zeta_{L}^{p}-\tau_{p / 3}$ and $\zeta_{T}^{p}-\tau_{p / 3}$ coincide with $p / 3$, showing that Eqs. (6) and (7) are valid.

A final comment is in order. In isotropic turbulence, the incompressibility condition says that second-order statistics are defined by a single scalar function. In fact, when we invoke $\mathrm{RSH}$ in the inertial range, it is easy to show that $\left\langle V_{T}^{2}\right\rangle /\left\langle V_{L}^{2}\right\rangle=$ $4 / 3$. We have indeed verified that this is quite accurately the case in the inertial range. Such relationships between higher-order moments of $V_{L}$ and $V_{T}$ are not straightforward, but computations suggest that they too approach, in the inertial range, constants whose numerical values increase with the order of the moment.

\section{CONCLUSION}

We have used DNS data at high Reynolds numbers to assess the past assertion that the longitudinal and transverse velocity structure functions scale differently in the inertial range. This difference was thought to be connected to the notion that the longitudinal velocity increments are connected to dissipation and the transverse velocity increments are connected to enstrophy, while the dissipation and enstrophy themselves have different PDFs. An implication is that the Kolmogorov RSH would have to be postulated independently, and differently, for the longitudinal and transverse velocity increments.

In Ref. [19], it has been shown, using the highest Reynolds numbers attained in the $8192^{3}$ simulations, that dissipation and enstrophy essentially scale the same and that any differences seen in the past are due to lower finite Reynolds numbers of the past simulations. It is then natural to revisit the previous work in some detail.

Using the high Reynolds number simulations of [19], we have shown that, in isotropic turbulence at high enough Reynolds numbers, the longitudinal and transverse velocity increments scale the same. Furthermore, a single RSH theory, which connects the statistics of velocity increments to the locally averaged dissipation rate, subsumes both longitudinal and transverse cases. That is, a single scaling group, $\delta u_{r}$ and $\epsilon_{r}$, with a single set of independent exponents, is enough to describe intermittent physics in fluid turbulence in the inertial range of isotropic turbulence.

These findings suggest the possibility of a deeper connection between the symmetric part of the strain rate, i.e., the dissipation physics, and the antisymmetric part of the strain rate, i.e., vortex dynamics, than previously portended. It is possible, however, that this deeper connection is violated in shear flows. We recall that, among the past data showing differences between $\zeta_{L}^{p}$ and $\zeta_{T}^{p}$ was a very high Reynolds number measurement in the atmospheric boundary layer [7]. We believe that it is the remnant shear effect in that flow that may have introduced some nontrivial aspects that break the deeper connection just mentioned.

\section{ACKNOWLEDGMENTS}

We thank Luca Biferale for useful discussions and D. Buaria and X. M. Zhai for their help in making data available. K.I. acknowledges funding from the European Research Council under the European Community's Seventh Framework Program, ERC Grant Agreement No. 339032. This work is partially supported by the National Science Foundation (NSF), via Grants No. CBET-1139037 and No. ACI-1036170 at the Georgia Institute of Technology. The computations were performed using supercomputing resources provided through the XSEDE consortium (which is funded by NSF) at the Texas Advanced Computing Center at the University of Texas (Austin), and the Blue Waters Project at the National Center for Supercomputing Applications at the University of Illinois (Urbana-Champaign).
[1] A. N. Kolmogorov, Dokl. Akad. Nauk. SSSR 30, 299 (1941a).

[2] A. N. Kolmogorov, Dokl. Akad. Nauk. SSSR 434, 16 (1941b).

[3] K. R. Sreenivasan and R. A. Antonia, Annu. Rev. Fluid Mech. 29, 435 (1997).

[4] A. S. Monin and A. M. Yaglom, Statistical Fluid Mechanics (MIT Press, Cambridge, MA, 1975), Vol. 2.

[5] U. Frisch, Turbulence (Cambridge University Press, Cambridge, UK, 1995).
[6] R. Camussi and R. Benzi, Phys. Fluids 9, 257 (1997).

[7] B. Dhruva, Y. Tsuji, and K. R. Sreenivasan, Phys. Rev. E 56, R4928 (1997).

[8] S. Chen, K. R. Sreenivasan, M. Nelkin, and N. Cao, Phys. Rev. Lett. 79, 2253 (1997).

[9] S. Grossmann, D. Lohse, and A. Reeh, Phys. Fluids 9, 3817 (1997). 
[10] R. Benzi, L. Biferale, R. Fisher, D. Q. Lamb, and F. Toschi, J. Fluid Mech. 653, 221 (2010).

[11] G. Gotoh, D. Fukayama, and T. Nakano, Phys. Fluids 14, 1065 (2002).

[12] T. Ishihara, T. Gotoh, and Y. Kaneda, Annu. Rev. Fluid Mech. 41, 165 (2009).

[13] R. Grauer, H. Homann, and J. F. Pinton, New J. Phys. 14, 063016 (2012).

[14] A. Noullez, G. Wallace, W. Lempert, R. B. Miles, and U. Frisch, J. Fluid Mech. 339, 287 (1997).

[15] D. A. Donzis, P. K. Yeung, and K. R. Sreenivasan, Phys. Fluids 20, 045108 (2008).

[16] V. L'vov and I. Procaccia, Phys. Fluids 8, 2565 (1996).

[17] M. Nelkin, Phys. Fluids 11, 2202 (1999).

[18] L. Biferale and I. Procaccia, Phys. Rep. 414, 43 (2005).

[19] P. K. Yeung, X. M. Zhai, and K. R. Sreenivasan, Proc. Natl. Acad. Sci. USA 112, 12633 (2015).

[20] A. N. Kolmogorov, J. Fluid Mech. 13, 82 (1962).
[21] D. A. Donzis and P. K. Yeung, Physica D 239, 1278 (2010).

[22] K. P. Iyer, K. R. Sreenivasan, and P. K. Yeung, Phys. Rev. E 92 , 063024 (2015).

[23] R. Benzi, S. Ciliberto, R. Tripiccione, C. Baudet, F. Massaioli, and S. Succi, Phys. Rev. E 48, R29(R) (1993).

[24] A. M. Oboukhov, J. Fluid Mech. 13, 77 (1962).

[25] S. T. Thoroddsen and C. W. Van Atta, Phys. Fluids A: Fluid Dyn. 4, 2592 (1992).

[26] A. A. Praskovsky, Phys. Fluids A: Fluid Dyn. 4, 2589 (1992).

[27] I. Hosokawa, C. W. Van Atta, and S. T. Thoroddsen, Fluid. Dyn. Res. 13, 329 (1994).

[28] G. Stolovitzky and K. R. Sreenivasan, Rev. Mod. Phys. 66, 229 (1994).

[29] G. Stolovitzky, P. Kailasnath, and K. R. Sreenivasan, Phys. Rev. Lett. 69, 1178 (1992).

[30] L. P. Wang, S. Chen, J. G. Brasseur, and J. C. Wyngaard, J. Fluid Mech. 309, 113 (1996). 